\title{
Photoluminescence enhancement of hexagonal-phase ZnS:Mn nanostructures using 1-thioglycolic acid
}

\author{
G. Murugadoss $\cdot$ V. Ramasamy $\cdot$ M. Rajesh Kumar
}

Received: 5 February 2013/Accepted: 27 March 2013/Published online: 9 April 2013

(c) The Author(s) 2013. This article is published with open access at Springerlink.com

\begin{abstract}
Hexagonal wurtzite $\mathrm{ZnS}: \mathrm{Mn}^{2+}$ nanostructures were synthesized at lower temperature $\left(80^{\circ} \mathrm{C}\right)$ by chemical method in an air atmosphere using 1-thioglycolic acid (TGA) as a stabilizing agent. It is a simple, highly efficient and energy-saving method for large-scale synthesis of hexagonal $\mathrm{ZnS}: \mathrm{Mn}^{2+}$ nanoparticles at lower temperature. Structural and optical properties of the samples were investigated. An optimum concentration of TGA was selected through optical PL study.
\end{abstract}

Keywords Nanoball - Nanorod - Surface defect .

TGA · Luminescence

\section{Introduction}

Zinc sulfide is one of the II-VI group compound mostly found in two structural polymorphs_cubic sphalerite or hexagonal wurtzite. It is a wide band gap material with unique properties in applications such as photocatalysts, solar cells, display panels, optical sensors and single electron transistors (Karar et al. 2004; Mu et al. 2005). It is the best host material for a large variety of dopants

\section{G. Murugadoss ( $\square)$}

Centre for Nanoscience and Technology, Anna University, Chennai 600 025, Tamilnadu, India

e-mail: murugadoss_g@yahoo.com

V. Ramasamy · M. Rajesh Kumar

Department of Physics, Annamalai University, Annamalai Nagar, Chidambaram 608 002, Tamilnadu, India
(Sooklal et al. 1996). Bhargava et al. (1994a, b) reported the first observation of optical properties of $\mathrm{Mn}^{2+}$ doped $\mathrm{ZnS}$ nanocrystals. It is well known that the cubic phase (zinc blende) of $\mathrm{ZnS}$ is stable at room temperature, while the hexagonal phase (wurtzite) is a high-temperature form. The equilibrium phase transformation between cubic and hexagonal phases occurs at about 1,296 K (Yu and Yoshimura 2002). Since the inherent crystal structures of $\mathrm{ZnS}$ have an important effect on its physical and chemical properties (Moore et al. 2004; Jiang et al. 2003), the preparation of $\mathrm{ZnS}$ with controllable phase is vital to its practical applications. Current research work is focused on nanosize particles and devices, because the particles display unique properties in the orange region and therefore have a lot of potential properties.

$\mathrm{Mn}^{2+}$ usually occupies substitutional sites in the $\mathrm{Zn}$ lattice as a divalent ion, and the excitation and decay of this ion produce an orange luminescence at approximately $590 \mathrm{~nm}$ (Bhargava et al. 1994a; Sapra et al. 2003). This emission peak is generally associated with a transition between ${ }^{4} \mathrm{~T}_{1}$ and ${ }^{6} \mathrm{~A}_{1}$ energy levels. In addition, electron paramagnetic resonance (EPR) technique has been widely used to obtain an insight into the local crystal field effects and symmetry around $\mathrm{Mn}^{2+}$ ions (Borse et al. 1999). The observed $\mathrm{Mn}^{2+}$ spectral features were used to explain the changes in the optical properties of the material. Manganese-doped $\mathrm{ZnS}$ nanoparticles have been synthesized using different methods such as laser ablation, certain physical methods, solution growth, and other different chemical techniques (Soo et al. 1994). In this study, hexagonal $\mathrm{ZnS}: \mathrm{Mn}^{2+}$ nanostructures were synthesized by chemical method using thioglycolic acid and its structural and photoluminescence properties were studied. It is a simple energy-saving method to synthesize hexagonal $\mathrm{ZnS}: \mathrm{Mn}^{2+}$ at lower temperatures.

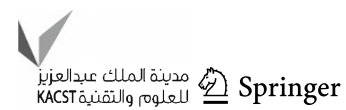




\section{Experimental}

Materials

To synthesize $\mathrm{Mn}$-doped $\mathrm{ZnS}$ nanoparticles, the following materials were used. Zinc acetate $\left(\mathrm{Zn}\left(\mathrm{CH}_{3} \mathrm{COO}\right)_{2} \cdot 2 \mathrm{H}_{2} \mathrm{O}\right)$, manganese chloride $\left(\mathrm{MnCl}_{2} \cdot 4 \mathrm{H}_{2} \mathrm{O}\right)$ and sodium sulfide $\left(\mathrm{Na}_{2} \mathrm{~S} \cdot x \mathrm{H}_{2} \mathrm{O}\right)$ obtained from Nice Chemical company were used as precursors. Thioglycolic acid (TGA) was obtained from Aldrich Company. The chemical reagents used were analytical reagent grade without further purification. All the glasswares used in this experimental work were acid washed. Ultrapure water was used for all dilutions and sample preparation.

Synthesis of Mn-doped $\mathrm{ZnS}$ nanoparticles

In a typical synthesis, $0.5 \mathrm{M}$ of $\mathrm{Zn}\left(\mathrm{CH}_{3} \mathrm{COO}\right)_{2} \cdot 2 \mathrm{H}_{2} \mathrm{O}$ in $50 \mathrm{ml}$ de-ionized water and different amounts of $(5-25 \mathrm{ml})$ thioglycolic acid (TGA) in $50 \mathrm{ml}$ de-ionized water were dissolved. Then, $4 \%$ of manganese chloride in $25 \mathrm{ml}$ was added. The optimum concentration of Mn was selected from the previous work (Murugadoss et al. 2010a). Subsequently, the $\mathrm{Na}_{2} \mathrm{~S}(50 \mathrm{ml})$ was added drop wise to the above mixture. For each experiment, the molar amounts of $\mathrm{Zn}\left(\mathrm{CH}_{3} \mathrm{COO}\right)_{2}$ and $\mathrm{Na}_{2} \mathrm{~S}$ used were equal. The $\mathrm{pH}$ value of the colloidal solution was reduced from 6 to 2 with respect to concentration of the TGA from 5, 20, 15, 20 and $25 \mathrm{ml}$, respectively. During the whole reaction process, the reactants were vigorously stirred under air atmosphere at $80{ }^{\circ} \mathrm{C}$. After $30 \mathrm{~min}$, the colloidal solution was kept for 1 week at room temperature. Then, the formed nanoparticles were separated from the colloidal solution by centrifuging. The collected precipitate was washed several times by de-ionized water and ethanol and then dried at $120{ }^{\circ} \mathrm{C}$ in an air atmosphere.

\section{Characterization}

The X-ray diffraction patterns of the products were recorded using $\mathrm{X}$-ray diffractometer (XRD, X'pert PRO) with $\mathrm{Cu} \mathrm{K}_{\alpha}$ radiation $(\lambda=1.54060 \AA)$ at room temperature. The crystallite size was estimated using the Scherrer's equation $D=(0.9 \lambda) /(\beta \cos \theta)$ from the full width at half maximum of the major XRD peak. The morphology and interior structure of the products were analyzed using TEM (Technai 20G2, FEI). The optical absorption spectra of the particles in de-ionized water were recorded using UV-1650PC SHIMADZU spectrophotometer. The photoluminescence measurements were performed on a RF5301PC spectrophotometer. Electron spin resonance (ESR) spectrum of the powder of the $\mathrm{ZnS}: \mathrm{Mn}^{2+}$ was measured on an ESR spectrometer (Bruker EMX Plus) at room temperature.

\section{Results and discussion}

Structural and morphological study

The crystal structures of the samples were investigated through the XRD study. Figure 1 shows the XRD pattern of the $\mathrm{ZnS}: \mathrm{Mn}^{2+}$ with capped TGA. The XRD pattern reveals the formation of perfectly indexed highly crystalline hexagonal wurtzite phase (JCPDS, card no. 80-0007) of $\mathrm{ZnS}: \mathrm{Mn}^{2+}$ nanoparticles. Previous research work (Murugadoss 2011), no observed hexagonal phase for Mn doped (up to $20 \%$ ) and surfactants capped ZnS:Mn (Biswas et al. 2005). However, a phase transition (cubic to hexagonal wurtzite) is detected only for the annealing sample ( $\mathrm{ZnS}: \mathrm{Mn}$ ) at higher temperatures (Biswas et al. 2005). The broadening of the diffraction peaks indicates the nanocrystalline nature of the sample. Actually, in the presence of the organic compound (TGA), it contains both a thiol (mercaptan) and a carboxylic acid, the free energy difference between the hexagonal and cubic phase might have increased toward their normal value, and as a result, the cubic phase gets favored over the hexagonal phase. This is the reverse of the reaction reported in the literature regarding the transformation of cubic $\mathrm{ZnS}$ to hexagonal wurtzite phase in the presence of some organic molecules (Murakoshi et al. 1998). However, an exact mechanism behind the formation of hexagonal nanorods by stabilizer is not clear and needs further study. Along with the main diffraction peaks some low-intensity impurity peaks are detected in Fig. 1. It may be due to the formation of $\mathrm{MnS}$ in the colloidal form.

The morphology of the samples was investigated through the transmission electron microscopic images (Fig. 2a-f). This reveals the formation of nanoballs and

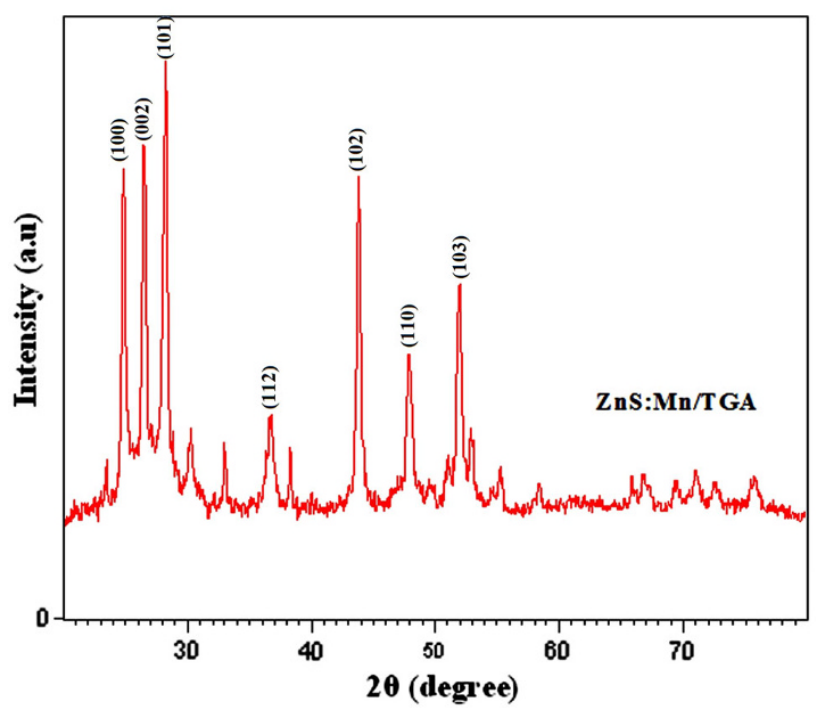

Fig. 1 X-ray diffraction analysis of TGA $(15 \mathrm{ml})$ capped $\mathrm{ZnS}: \mathrm{Mn}^{2+}$ nanoparticles 
Fig. 2 a-e TEM micrographs of TGA capped $\mathrm{ZnS}: \mathrm{Mn}^{2+}$ nanoparticles with different magnification and SAED pattern (f)
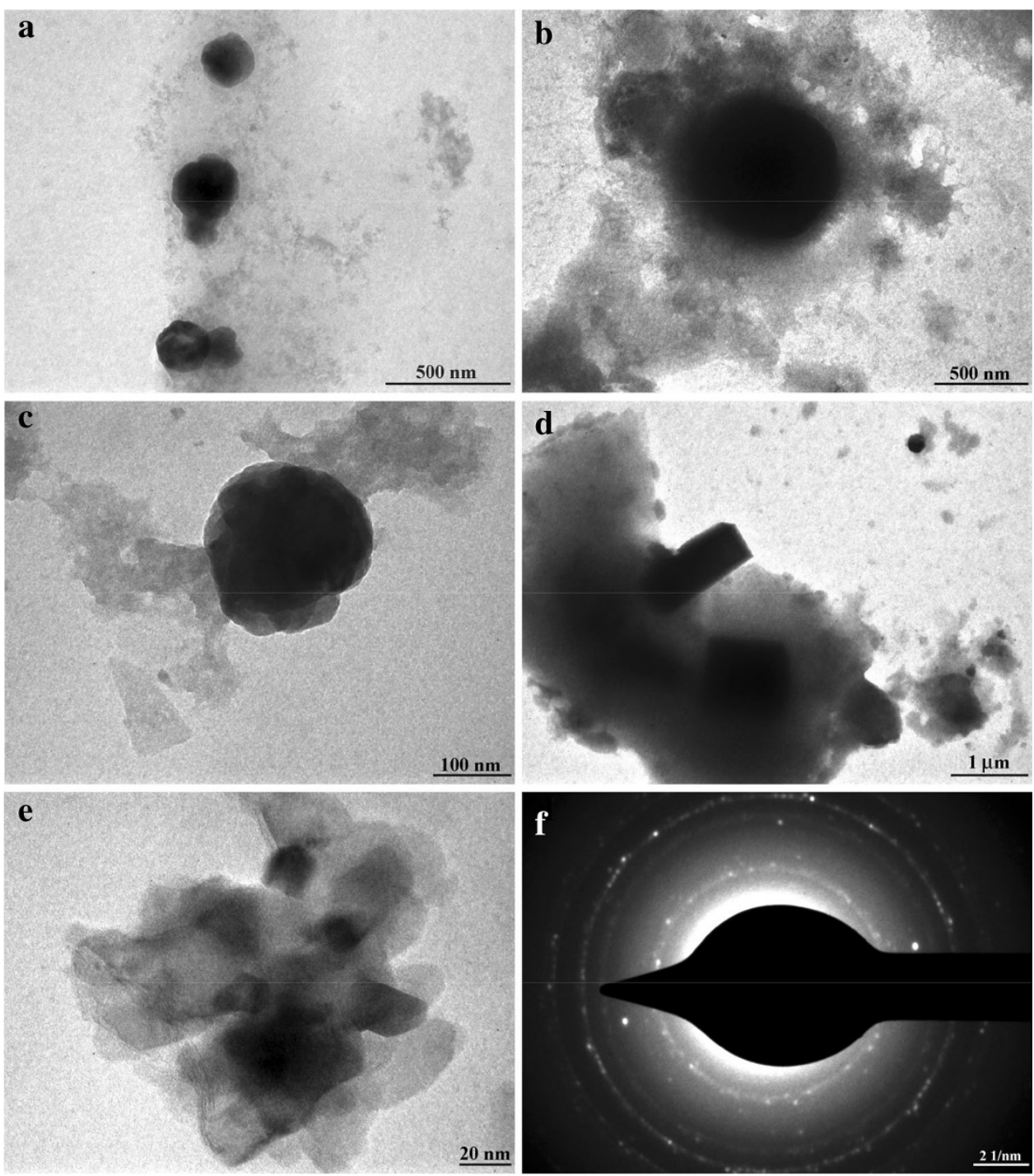

nanorods along with very tiny particles. The formation of complex structure (nanoballs and rods) may be due to the presence of TGA. At the suitable temperature $\left(80^{\circ} \mathrm{C}\right)$ the thiol and carboxylic molecules come out of the layered complex structure, resulting in the aggregation or assemblage by the seeds (very small size nanoparticles) as shown in Fig. 2. Figure 2a, $b$ shows the formation of the nanoballs $(\sim 100 \mathrm{~nm})$ by the assemblage of very small size nanoparticles $(<5 \mathrm{~nm})$. The sizes of the nanoballs varied within $100-150 \mathrm{~nm}$ range. Figure 2c shows a complete formation of the isolated nanoball. Well-shaped hexagonal structured ZnS:Mn (Fig. 2d, e) nanorods were also obtained along with nanoballs and very small size particles. It may be because of the stabilization of the synthesized particles in the colloidal form for long time (for 15 days) with the presence of the TGA. Additionally, it should be pointed out that the tiny size of as-synthesized $\mathrm{ZnS}$ particles made it easy for cubic $\mathrm{ZnS}$ to transform into hexagonal $\mathrm{ZnS}$ at low temperatures (Qadri et al. 1999). Consequently, the capping agent TGA can be used as a simple, highly efficient, and energy saving method for large-scale synthesis of hexagonal $\mathrm{ZnS}: \mathrm{Mn}$ nanoparticles.The diameters and lengths of the nanorods varied within $50-100 \mathrm{~nm}$ and $1-1.5 \mu \mathrm{m}$, respectively. In addition, very small size (>20 nm dia.) nanorods are detected (Fig. 2e). Corresponding SAED pattern of the sample is shown in Fig. $2 \mathrm{f}$.

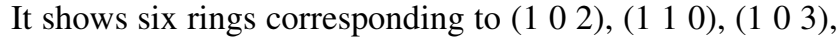
$\left(\begin{array}{lll}2 & 0 & 0\end{array}\right),\left(\begin{array}{lll}1 & 1 & 2\end{array}\right)$ and $\left(\begin{array}{lll}2 & 0 & 1\end{array}\right)$ phases of the hexagonal structure.

\section{Optical study}

UV-Visible spectrophotometry is one of the basic techniques to study the quantum confinement effects. Figure 3 
shows the UV-Visible absorption spectra of the $\mathrm{ZnS}: \mathrm{Mn}^{2+}$ (4\%) nanoparticles stabilized by thioglycolic acid (5$25 \mathrm{ml}$ ). The characteristic absorption peaks of the samples were appeared in the range $250-350 \mathrm{~nm}$. The peak position reflects the band gap of the nanoparticles. The obtained

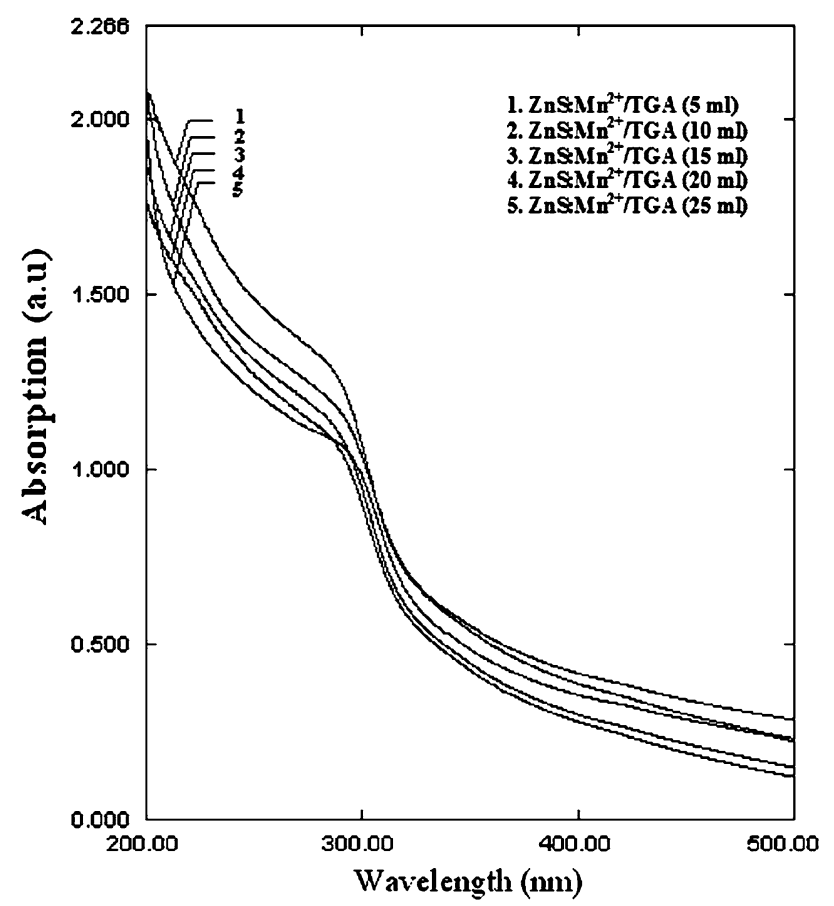

Fig. 3 UV-Visible absorption spectra of TGA capped (5-25 ml) $\mathrm{ZnS}: \mathrm{Mn}^{2+}$ nanoparticles

Fig. 4 a PL spectra of TGA capped $(5-25 \mathrm{ml}) \mathrm{ZnS}: \mathrm{Mn}^{2+}$ nanoparticles and b corresponding concentration versus PL intensity peaks are centered at $292 \mathrm{~nm}$. As seen in Fig. 3, the peak position has no change with respect to the TGA concentrations. However, a strong absorption is observed for $5 \mathrm{ml}$ of TGA. The absorption peaks are significantly blue-shifted from the bulk $\mathrm{ZnS}$ (345 nm). It dictates the reduction of the particles size in the nanoscale. This shifting is due to quantum confinement effect (Deshpande et al. 2009). It is well known that the fundamental absorption can be used to determine the nature and value of the optical band gap of the nanoparticles. The band gap of all the samples is calculated using a simple wave-energy equation:

$E_{\mathrm{gn}}=h v=h c / \lambda$

where $h$ is the Planck's constant, $c$ is the light velocity and $\lambda$ is the absorption wavelength. From the band gap value, the size $(R)$ of nanoparticles can be calculated by the Brus model (Brus 1991)

$$
\begin{aligned}
E_{g}(\text { nano })-E_{g}(\text { bulk })= & h^{2} / 8 R^{2}\left(1 / m_{\mathrm{e}}+1 / m_{\mathrm{h}}\right) \\
& -1.8 e^{2} /\left(4 \pi \varepsilon_{\alpha} \varepsilon_{0} R\right)
\end{aligned}
$$

where $E_{\text {bulk }}$ and $E_{\text {nano }}$ are the band gap energies of bulk and nanosized semiconductors, respectively, $h$ is the Planck's constant, $m_{\mathrm{e}}$ and $m_{\mathrm{h}}$ are the effective masses of electron and hole, respectively, $\varepsilon_{\alpha}$ is the dielectric constants of the material $\left(\varepsilon_{\alpha}=8.3\right)$ and $\varepsilon_{0}$ is the permittivity of vacuum. Here, $m_{\mathrm{e}}=0.19 \mathrm{~m}_{0}$ and $m_{\mathrm{h}}=0.8 \mathrm{~m}_{0}$, where $m_{0}$ is the free electron mass and $e$ is the charge of electron (in coulomb). The calculated particles size is $3.7 \mathrm{~nm}$.

Figure 4a depicts the PL spectra of the $\mathrm{ZnS}: \mathrm{Mn}^{2+}$ nanoparticles stabilized by different amounts $(5-25 \mathrm{ml})$ of
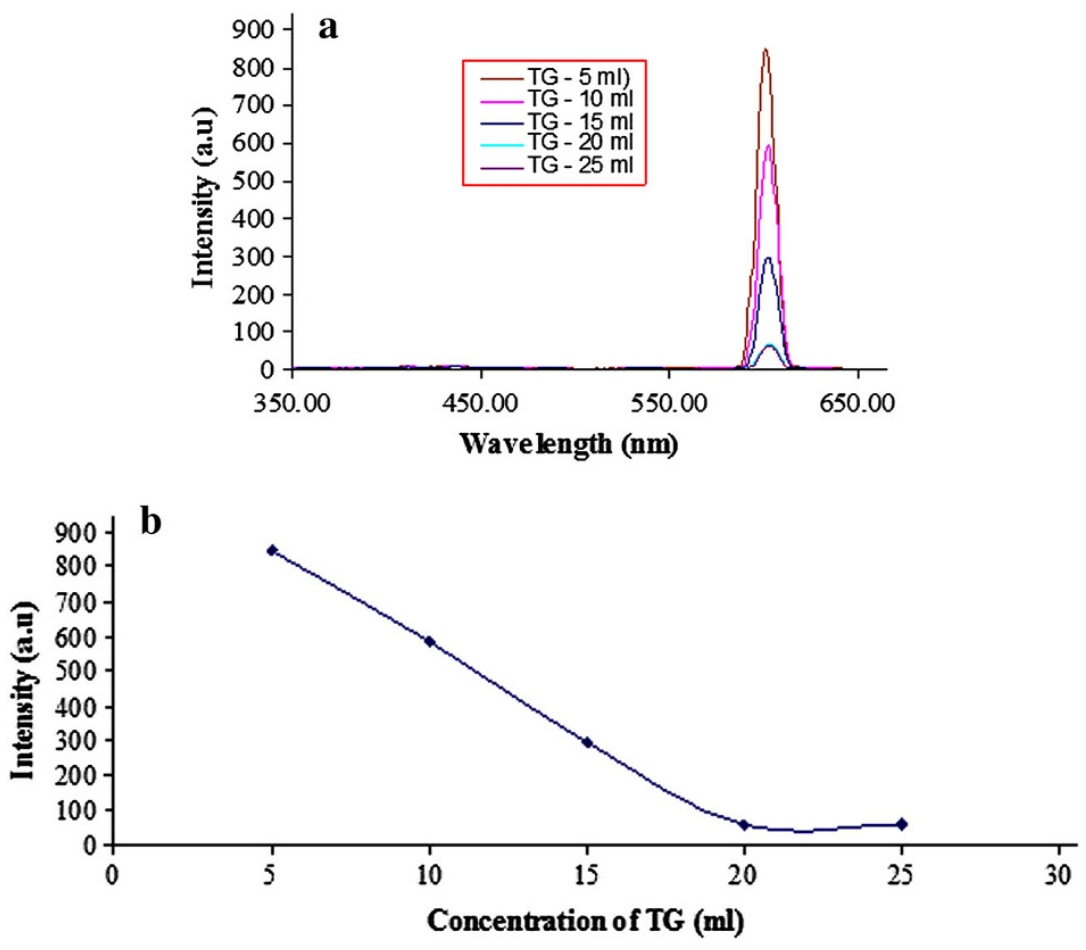
TGA. The PL spectra show two emission bands, one is the strong orange emission band located at about $580 \mathrm{~nm}$ from intraconfigurational $\mathrm{d}-\mathrm{d}$ transitions ${ }^{4} \mathrm{~T}_{1}-{ }^{6} \mathrm{~A}_{1}$ in $\mathrm{T}_{\mathrm{d}}$ symmetry which are in principle spin forbidden by the $S=0$ and parity selection rules; the other is a weak and broad blue band centered at about $445 \mathrm{~nm}$, which is considered to be associated with defects (Murugadoss et al. 2010b). The narrow orange emission indicates the particles are highly homogeneous. Figure $4 \mathrm{~b}$ shows a plot between the concentrations of TGA versus PL intensity. As seen in Fig. 4b, a maximum PL intensity is observed for lower concentration of stabilizer (TGA, at $5 \mathrm{ml}$ ), and after that, it is decreased.

At higher concentrations of TGA $(>5 \mathrm{ml})$, the $\mathrm{pH}$ of the colloidal solution is suddenly reduced $(<4)$. Hence, the PL intensity of $\mathrm{ZnS}: \mathrm{Mn}$ is significantly decreased. It dictates that the increase of the surface defect or decomposition of the sample is due to increase of the carboxylic acid by increasing TGA concentration. The $\mathrm{ZnS}: \mathrm{Mn} / \mathrm{TGA}$ ( $5 \mathrm{ml}$ ) gave an enhanced PL efficiency [the ratio of intensity of the orange emission $\left(I_{1}\right)$ to blue emission $\left(I_{0}\right)$ ] as shown in Fig. 4b. The increasing efficiency indicates the improvement of homogeneous $\mathrm{ZnS}: \mathrm{Mn}^{2+}$ growth and declining surface defect. The role of the stabilizer is not only to tailor crystalline structure but also to control the size in the nanoscale. In addition, the PL enhancement indicates elimination of the surface defect completely by TGA.

\section{ESR study}

The presence of $\mathrm{Mn}^{2+}$ ions in $\mathrm{ZnS}$ lattice nanostructure can be confirmed by ESR spectrum. The ESR spectrum (Fig. 5) of $\mathrm{ZnS}: \mathrm{Mn}^{2+}$ nanostructure shows six lines which is attributed to $\left(\Delta M_{I}=0\right.$ and $\left.\Delta M_{S}= \pm 1\right)$ allowed transitions of the $\mathrm{Mn}^{2+}$ ions $(I=5 / 2)$. For lower Mn concentrations,

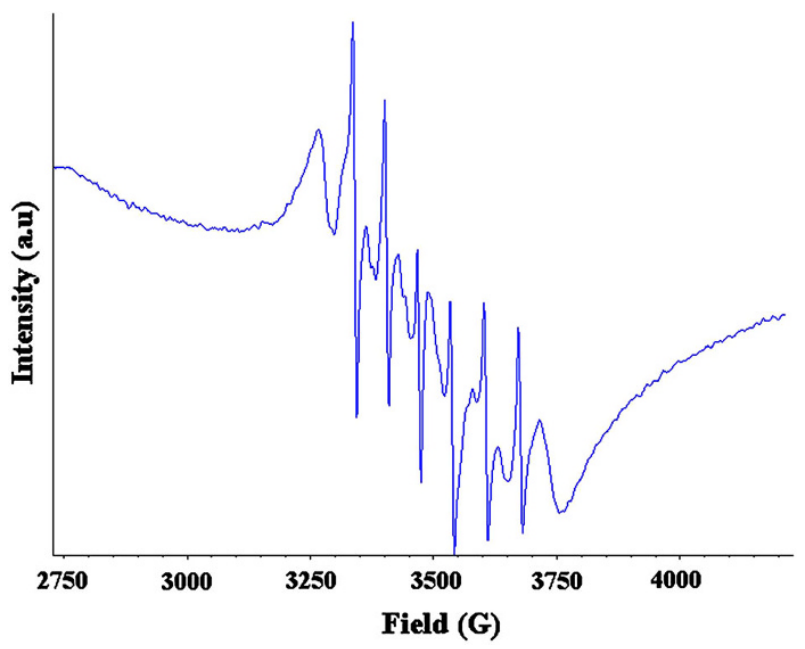

Fig. 5 ESR spectrum of $\mathrm{ZnS}: \mathrm{Mn}^{2+}(5 \mathrm{ml})$ nanoparticles these lines are accompanied by smaller ones between them which are assigned to $\left(\Delta M_{I}=0\right.$ and $\left.\Delta M_{S}= \pm 1\right)$ forbidden transitions. The appearance of the well-resolved hyperfine structure together with the less intense lines between them (at approximately $g=2$ ) proves that many $\mathrm{Mn}$ ions are at nearly cubic symmetry (Sooklal et al. 1996; Hoffman et al. 1998). As the $\mathrm{Mn}^{2+}$ ion is affected by other $\mathrm{Mn}^{2+}$ ions that are randomly distributed around it, the Mn-Mn interactions should result in a reduction of the electron spin-nuclear spin interactions in an individual Mn ion (Ji et al. 2003).

\section{Conclusions}

In summary, the hexagonal wurtzite $\mathrm{ZnS}: \mathrm{Mn}^{2+}$ nanostructures (nanoballs and nanorods) were successfully synthesized by simple chemical method and studied using XRD, TEM, UV-Vis and PL. It is a simple, highly efficient and energy-saving method for large-scale synthesis of hexagonal $\mathrm{ZnS}: \mathrm{Mn}^{2+}$ nanoparticles at lower temperature. The experimental results demonstrated that as-prepared $\mathrm{ZnS}: \mathrm{Mn}^{2+} / \mathrm{TGA}$ nanostructures have an excellent optical property. An optimum concentration of the TGA was selected as $5 \mathrm{ml}$ (since it shows enhanced PL intensity) and it tailored the structure of the $\mathrm{ZnS}: \mathrm{Mn}^{2+}$. The presence of $\mathrm{Mn}^{2+}$ ions was confirmed by ESR study. The intense emission was observed for Mn-doped $\mathrm{ZnS}$ nanoparticles using TGA, which will be useful for fabrication of optoelectronic devices.

Open Access This article is distributed under the terms of the Creative Commons Attribution License which permits any use, distribution, and reproduction in any medium, provided the original author(s) and the source are credited.

\section{References}

Bhargava RN, Gallagher D, Hong X, Nurmikko A (1994a) Optical properties of manganese-doped nanocrystals of ZnS. Phys Rev Lett 72:416-419

Bhargava RN, Gallagher D, Welker T (1994b) Doped nanocrystals of semiconductors-a new class of luminescent materials. J Lumin 60-61:275-280

Biswas S, Kar S, Chaudhuri S (2005) Optical and magnetic properties of manganese-incorporated zinc sulfide nanorods synthesized by a solvothermal process. J Phys Chem B 109:17526-17530

Borse PH, Srinivas D, Shinde RF, Date SK, Vogel W, Kulkarni SK (1999) Effect of $\mathrm{Mn}^{2+}$ concentration in $\mathrm{ZnS}$ nanoparticles on photoluminescence and electron-spin-resonance spectra. Phys Rev B 60:8659-8664

Brus LE (1991) Quantum crystallites and nonlinear optics. Appl Phys A 53:465-474

Deshpande A, Shah P, Gholap RS, Gupta NM (2009) Interfacial and physico-chemical properties of polymer-supported CdS-ZnS nanocomposites and their role in the visible-light mediated photocatalytic splitting of water. J Colloid Interface Sci 333:263-268 
Hoffman DM, Hofstaetter A, Lei U, Meyer BK, Gounio G (1998) EPR and ENDOR investigations on CdS:Mn nanocrystals. J Cryst Growth 184(185):383-387

Ji TH, Jain WB, Fang JY (2003) The first synthesis of $\mathrm{Pb}_{1-x} \mathrm{Mn}_{x} \mathrm{Se}$ nanocrystals. J Am Chem Soc 125:8448-8449

Jiang Y, Meng XM, Liu J, Hong ZR, Lee CS, Lee ST (2003) ZnS nanowires with wurtzite polytype modulated structure. Adv Mater 15:1195-1198

Karar N, Raj S, Singh F (2004) Properties of nanocrystalline ZnS:Mn. J Cryst Growth 268:585-589

Moore D, Ronning C, Ma C, Wang ZL (2004) Wurtzite ZnS nanosaws produced by polar surfaces. Chem Phys Lett 385:8-11

$\mathrm{Mu} \mathrm{J}, \mathrm{Gu} \mathrm{D}, \mathrm{Xu} \mathrm{Z}$ (2005) Effect of annealing on the structural and optical properties of non-coated and silica-coated $\mathrm{ZnS}: \mathrm{Mn}$ nanoparticles. Mater Res Bull 40:2198-2204

Murakoshi K, Hosokawa H, Tanaka N, Saito M, Wada Y, Sakata T, Mori H, Yanagida S (1998) Phase transition of ZnS nanocrystallites induced by surface modification at ambient temperature and pressure confirmed by electron diffraction. Chem Commun 1998:321-322

Murugadoss G (2011) Synthesis, optical, structural and thermal characterization of $\mathrm{Mn}^{2+}$ doped $\mathrm{ZnS}$ nanoparticles using reverse micelle method. J Lumin 131:2216-2223
Murugadoss G, Rajamannan B, Ramasamy V (2010a) Synthesis, characterization and optical properties of water-soluble ZnS:Mn ${ }^{2+}$ nanoparticles. J Lumin 130:2032-2039

Murugadoss G, Rajamannan B, Ramasamy V (2010b) Synthesis and photoluminescence study of PVA-capped $\mathrm{ZnS}: \mathrm{Mn}^{2+}$ nanoparticles. Dig J Nanomater Biostruct 5:339-345

Qadri SB, Skelton EF, Hsu D, Dinsmore AD, Yang J, Gray HF, Ratna BR (1999) Size-induced transition-temperature reduction in nanoparticles of ZnS. Phys Rev B 60:9191-9193

Sapra S, Nanda J, Anand A, Bhat SV, Sarma DD (2003) Optical and magnetic properties of manganese-doped zinc sulfide nanoclusters. J Nanosci Nanotechnol 3:392-400

Soo YL, Ming ZH, Huang SW, Kao YH, Bhargava RN, Gallagher D (1994) Local structures around Mn luminescent centers in Mndoped nanocrystals of ZnS. Phys Rev B 50:7602-7607

Sooklal K, Cullum BS, Angel SM, Murphy CJ (1996) Photophysical properties of $\mathrm{ZnS}$ nanoclusters with spatially localized $\mathrm{Mn}^{2+}$. J Phys Chem 100:4551-4555

Yu SH, Yoshimura M (2002) Shape and phase control of ZnS nanocrystals: template fabrication of wurtzite $\mathrm{ZnS}$ single-crystal nanosheets and $\mathrm{ZnO}$ flake-like dendrites from a lamellar molecular precursor $\mathrm{ZnS} \cdot\left(\mathrm{NH}_{2} \mathrm{CH}_{2} \mathrm{CH}_{2} \mathrm{NH}_{2}\right)_{0.5}$. $\mathrm{Adv}$ Mater $14: 296-300$ 